\title{
Malassezia furfur Infection
}

National Cancer Institute

\section{Source}

National Cancer Institute. Malassezia furfur Infection. NCI Thesaurus. Code C82981.

A yeast infection usually manifested as a superficial skin infection. It may also present as a systemic infection in patients who are receiving total parenteral nutrition. 